\title{
From electronic structure to catalytic activity: A single descriptor for adsorption and reactivity on transition-metal carbides
}

\author{
A. Vojvodic, ${ }^{1, \text { * }}$ A. Hellman, ${ }^{1,2}$ C. Ruberto,${ }^{1}$ and B. I. Lundqvist ${ }^{1,3}$ \\ ${ }^{1}$ Department of Applied Physics, Chalmers University of Technology, SE-412 96 Göteborg, Sweden \\ ${ }^{2}$ Competence Centre for Catalysis, Chalmers University of Technology, SE-412 96 Göteborg, Sweden \\ ${ }^{3}$ Center for Atomic-scale Materials Design, Department of Physics, \\ Technical University of Denmark, DK-2800 Kgs. Lyngby, Denmark
}

(Dated: October 29, 2018)

\begin{abstract}
Adsorption and catalytic properties of the polar (111) surface of transition-metal carbides (TMC's) are investigated by density-functional theory. Atomic and molecular adsorption are rationalized with the concerted-coupling model, in which two types of TMC surface resonances (SR's) play key roles. The transition-metal derived SR is found to be a single measurable descriptor for the adsorption processes, implying that the Brønsted-Evans-Polanyi relation and scaling relations apply. This gives a picture with implications for ligand and vacancy effects and which has a potential for a broad screening procedure for heterogeneous catalysts.

PACS numbers: 68.43.Bc, 73.20.At, 73.20.-r
\end{abstract}

Finding new simple, efficient, and cheap catalysts is one way to solve some of today's global environmental challenges [1], as illustrated by a recent way to harvest solar energy [2]. A goal in catalysis research is to design and tune the activity and selectivity of catalysts by controlling their structural properties at the atomic level. This calls for an identification of key concepts, which can be done from fundamental theory, in particular densityfunctional theory (DFT), as shown for transition-metal (TM) surfaces [3].

For such surfaces, the important role of the TM $d$ electrons for chemisorption and catalysis was early anticipated [4], estimated [5], and articulated in the $d$-band model [3, 6], which establishes relations between atomic structure and activity for TM catalysts [7]. The $d$-band model explains and predicts a variety of TM properties, for example, adsorption energies on TM alloys, strengths of bonds at steps and terraces, and transition-state energies [3, 7], by correlating them to the energy of the $d$-band center. The success of the $d$-band model has led to the introduction of simple descriptors that are able to rationalize experimental data [3]. Examples of such descriptors, e.g., for the water gas shift reaction, are the adsorption energies of oxygen and carbon monoxide on TM surfaces. Today, design of new TM catalysts by computational screening is a realistic approach [8, 9].

A need for catalysts, a scarcity of precious catalyst materials, and a general curiosity spur the interest in catalyst materials beyond the TM's. Statements like "for several types of reactions, such as hydrogenation reactions, catalytic activities of carbides and nitrides were approaching or surpassing those of noble metals" can be found in the literature [10]. Thus, a theoretical approach extended to more complex materials than TM's is of interest. In a study of $\mathrm{H}$ adsorption on TM-terminated (111) surfaces of TM carbides (TMC's), a deviation from the $d$-band model has been observed [11]. A recent study of TM oxides, nitrides, and sulfides stresses similarities in scaling behavior between molecular and atomic adsorption energies but calls for "a suitably modified $d$-band model" [12]. There exist suggestions of a bulk-derived descriptor for the reactivity of the TMC's [13], however, our work goes beyond and finds a surface-derived descriptor based on electronic-structure calculations.

Electronic surface states and resonances (SR's), known to appear at steps and defects, play an important role for the catalytic activity of a system. The ideal and stable TMC(100) faces are common objects of study in the literature [14] and do not show any presence of SR's. Recently we have shown that the understanding of atomic adsorption on $\mathrm{TiC}$ and $\mathrm{TiN}$ calls for substantial steps beyond the original $d$-band model [15, 16, 17]. On $\operatorname{TiC}(111)$ and TiN(111), the key actors are identified to be two types of surface resonances (SR's), derived from Ti (TiSR) and $\mathrm{C}(\mathrm{N})$ states (CSR/NSR), respectively. The adsorption mechanism is explained within a concerted-coupling model (CCM), in which there are two types of interactions involved, one between the adsorbate and the TiSR and one between the adsorbate and the CSR's (NSR's) 15, 16, 17]. The presence of SR's motivates a focus on the metastable and polar TMC(111) surfaces, which may, for example, serve as prototypes for steps and defects.

This Letter demonstrates that an approach that involves DFT calculations, trend studies, analyses of computed results in electron-structural terms, formulation of a model, and utilization of model predictions [15, 16, 17] is useful to reach the above stated goal. In terms of the CCM, we identify a single descriptor, the mean energy of the TM-localized SR (TMSR), that accounts for three important processes on the considered TMC surfaces: adsorption, dissociation, and catalytic activity. The results should be general but are here illustrated for one surface reaction, the ammonia synthesis. As a consequence of the single descriptor, calculable and experimentally available 
for some substrates [18], scaling relations and BrønstedEvans-Polanyi (BEP) relations are shown to apply, in the same way as for TM surfaces [3]. Finally, the generality and practical usefulness of the conceptual picture is demonstrated by applications to ligand and defect effects, which also indicate possibilities for further refinements. Taken together, these results provide a framework for a systematic analysis of the catalytic activity of the TMC's.

Trend studies are conducted for the catalysis-relevant adsorbates $\mathrm{H}, \mathrm{N}, \mathrm{O}$, and $\mathrm{NH}_{x}(x=1,2,3)$, with respect to the TM component of the substrate TMC, spanning three different periods and four different groups in the periodic table $(\mathrm{TM}=\mathrm{Sc}, \mathrm{Ti}, \mathrm{V}, \mathrm{Zr}, \mathrm{Nb}, \mathrm{Mo}$, Ta, and $\mathrm{W})$ [19]. The first-principles study is performed within the densityfunctional theory (DFT), as implemented in DACAPO 20, 21]. The computational procedures are favorably tested and compared with literature data [23]. From the DFT calculations, the adsorption is described in terms of adsorption energies $\left(E_{\text {ads }}\right)$ and atom-projected local densities of states (LDOS).

Calculated total DOS and LDOS's for the TMterminated TMC(111) surfaces (illustrated for some representative cases in Fig. (1) reveal the existence of SR's on all surfaces. There is a TMSR in the vicinity of the Fermi energy $\left(E_{\mathrm{F}}\right)$ and there are several carbon-localized SR's (CSR's) deeper down in the valence band [24]. Note that $\mathrm{ScC}(111)$ has no filled metallic $d$ SR states.

The TMSR's are characterized by studying the difference in bulk and surface DOS's 24], where they appear as positive peaks owing to the build up of states at the surface. A parameter $\varepsilon_{\mathrm{CCM}}$ is defined as the mean energy (center of gravity) of the TMSR, in close analogy with the $d$-band center $\varepsilon_{\mathrm{d}}$ in the $d$-band model. The integration is performed over the energy range of the positive TMSR peak. The value of $\varepsilon_{\mathrm{CCM}}$ decreases as the group number of the TM component increases (Fig. 1), as expected from the filling of the TM $d$ states [24].

Analysis of the DOS before and after adsorption (illustrated for $\mathrm{O} / \mathrm{VC}$ in the lower panel of Fig. (1) shows that the TMSR is depleted upon adsorption. This supports the CCM assumption that only the surface-localized part of the $d$-band spectrum is relevant for the adsorption and that the TMSR's are key actors in the adsorbatesurface interaction [24]. Hence, $\varepsilon_{\mathrm{CCM}}$ is a descriptor for the atomic adsorption on TMC's. An exception is $\mathrm{ScC}$, with its empty TMSR and consequently an adsorption mainly arising from the interaction with the CSR's 24]. This exception confirms the presence of two types of interactions, as formulated in the CCM [15, 16, 17, 24].

The role of $\varepsilon_{\mathrm{CCM}}$ as a descriptor is confirmed by extensive DFT calculations, which yield a linear correlation between the $E_{\text {ads }}$ and $\varepsilon_{\mathrm{CCM}}$ values for each of the studied adsorbates (Fig. 2). The $E_{\text {ads }}$ value decreases (i.e., the adsorption gets stronger) as $\varepsilon_{\mathrm{CCM}}$ increases, with a gradient that varies strongly between different adsorbates. The slopes of the $E_{\text {ads }} v s . \varepsilon_{\mathrm{CCM}}$ correlations are steeper

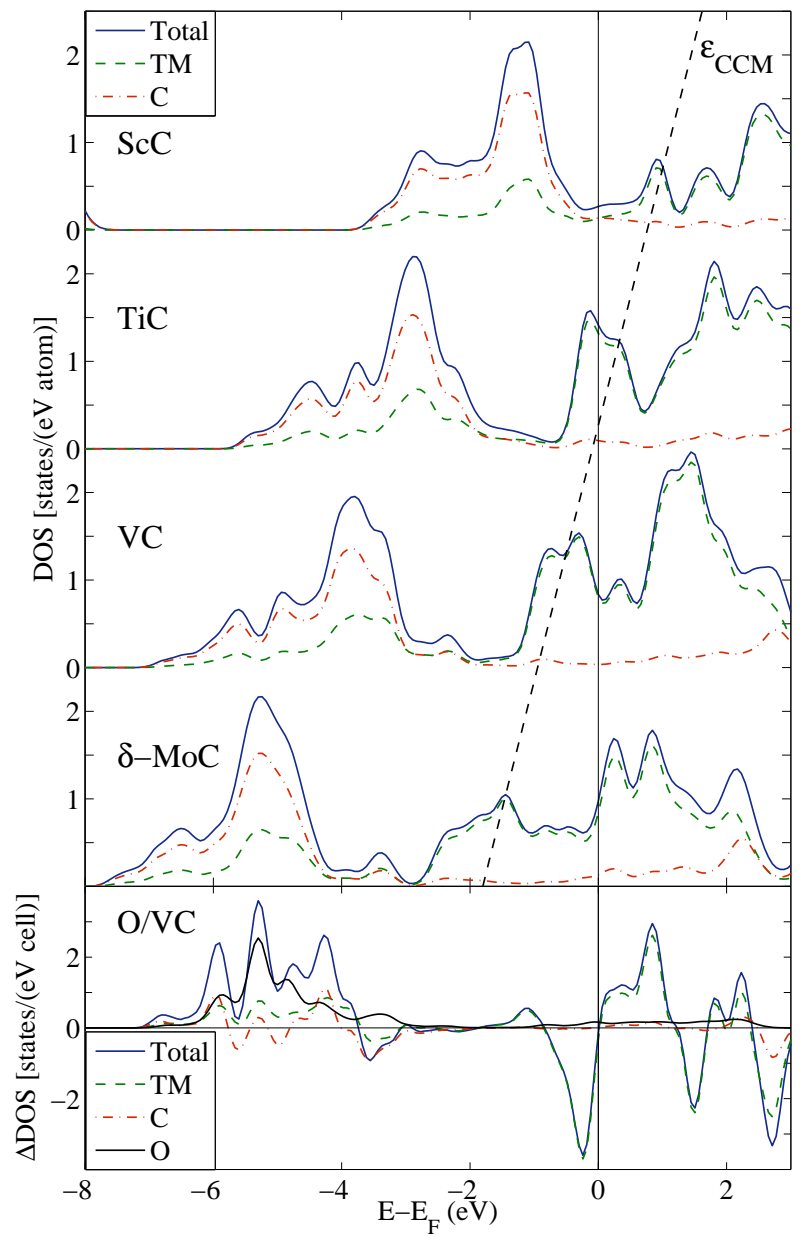

FIG. 1: Upper panel: Calculated atom-projected and total (111) surface DOS's for a group of TMC's. The dashed diagonal line connects the mean energies $\varepsilon_{\mathrm{CCM}}$ of the TMSR's and illustrates the downward shift of the TMSR energy along the TMC series as the group number of the TM component increases. Lower panel: Difference in surface DOS induced by $\mathrm{O}$ adsorption on $\mathrm{VC}(111)$.

for the TMC(111) surfaces than for TM's [3]. From a design perspective, this could be a useful property, allowing larger effects from small changes. Again, $\mathrm{ScC}$ does not follow the trend of the other TMC's, reflecting the different nature of its interaction with the adsorbate.

To further test that proper key concepts (TMSR and $\left.\varepsilon_{\mathrm{CCM}}\right)$ have been identified, ligand effects are studied. Manipulating the local environment of the surface TM atom changes $\varepsilon_{\mathrm{CCM}}$, thereby changing adsorption, activation, and other energies, which opens up for optimization of the catalytically active site. In our illustrative example, $\mathrm{O}$ adsorption on a $\mathrm{TiC}(111)$ surface with one, two, or three surface Ti atoms next to the fcc adsorption site replaced by $\mathrm{V}$ atoms, the calculated adsorption strength is successively reduced at the same time as the local value of $\varepsilon_{\mathrm{CCM}}$ is shifted down. This is expected in the CCM 


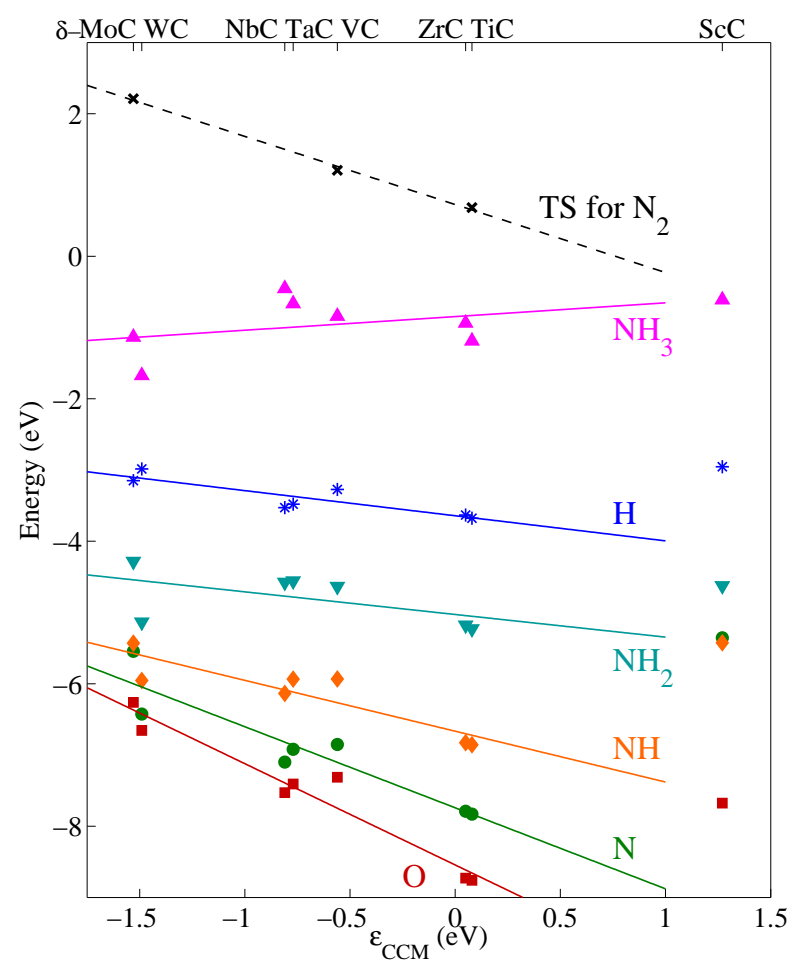

FIG. 2: Adsorption energies of $\mathrm{H}, \mathrm{N}, \mathrm{O}$, and $\mathrm{NH}_{x}(x=1,2,3)$ on the considered TMC(111) surfaces vs. the descriptor $\varepsilon_{\mathrm{CCM}}$ (the TMSR center of mass). The adsorption energy is defined as $E_{\mathrm{ads}}^{\mathrm{A}}=E_{\mathrm{TMC}+\mathrm{A}}-E_{\mathrm{TMC}}-E_{\mathrm{A}}$ for each adsorbate A. ( $\mathrm{ScC}$ is not included in the linear regressions). Also included (dashed line) are the transition-state (TS) energies for $\mathrm{N}_{2}$ dissociation, calculated with a fixed bond-length method.

(exchange of Ti with $\mathrm{V}$ shifts $d$ levels down in energy) and monitored by analyzing our calculated LDOS's also in these cases.

However, there is a deviation from the linear $E_{\text {ads }} v s$. $\varepsilon_{\mathrm{CCM}}$ relation [25]. For example, the $E_{\mathrm{ads}}$ value for the system with three neighboring $\mathrm{V}$ atoms on the $\mathrm{TiC}(111)$ surface is similar to that for pure $\mathrm{VC}(111)$, although their $\varepsilon_{\mathrm{CCM}}$ values differ. This ligand calculation points at further possible refinements of the descriptor. An orbital projected DOS shows that the main interaction takes place between the adsorbate and the $d_{x z+y z}$ components of the TMSR (the $z$ direction being perpendicular to the surface). Hence a refined descriptor, taken as the center of mass of these levels, could be introduced. However, such a detailed descriptor level might not be practical from an experimental point of view.

As $\varepsilon_{\mathrm{CCM}}$ is a descriptor for both atomic and molecular adsorption (Fig. 2), there is a scaling relation between the molecular and atomic $E_{\text {ads }}$ values (Fig. 3). Similar scaling relations have been found for several molecular species on TM's [26] and for $\mathrm{OH}_{\mathrm{x}}$ on TM oxides, $\mathrm{NH}_{\mathrm{x}}$

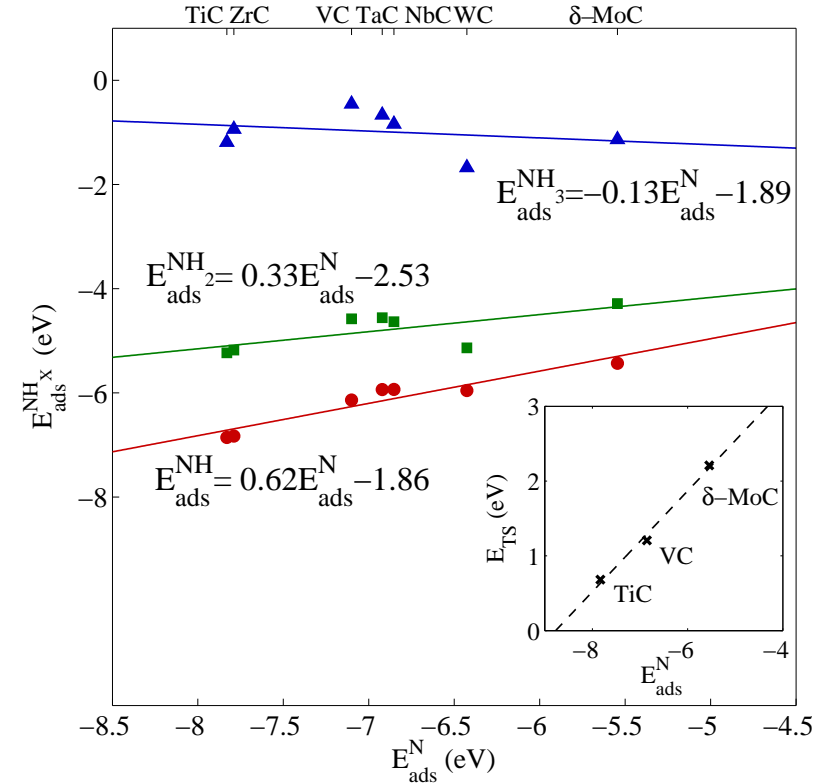

FIG. 3: Adsorption energies on TMC(111) surfaces ( $\mathrm{ScC}$ is not included $)$ for $\mathrm{NH}_{x}(\mathrm{x}=1,2,3)$ vs. $\mathrm{N}$ atomic adsorption energies (scaling relation). The adsorption energy is defined as $E_{\text {ads }}^{\mathrm{NH}_{x}}=E_{\mathrm{TMC}+\mathrm{NH}_{x}}-E_{\mathrm{TMC}}-E_{\mathrm{NH}_{x}}$. Inset: the linear variation of the activation energy for $\mathrm{N}_{2}$ dissociation $\left(E_{\mathrm{TS}}\right)$ vs. $\mathrm{N}$ atomic adsorption energy on $\mathrm{TiC}, \mathrm{VC}$, and $\delta$-MoC (BEP relation).

on TM nitrides, and $\mathrm{SH}_{\mathrm{x}}$ on TM sulfides [12]. These scaling relations can be described by a linear equation $E_{\mathrm{ads}}^{\mathrm{AH}_{x}}=\gamma(x) E_{\mathrm{ads}}^{\mathrm{A}}+\xi$, where $\gamma(x)$ depends only on the number $x$ of $\mathrm{H}$ atoms in the molecule [3]. Our results (Fig. 3) yield values $\left(\gamma_{\mathrm{NH}}=0.62, \gamma_{\mathrm{NH}_{2}}=0.33, \gamma_{\mathrm{NH}_{3}}=\right.$ -0.13 ) that are close to the predictions $2 / 3,1 / 3$, and 0 for $x=1,2$, and 3 , respectively. The small deviations are likely to be caused by the $\mathrm{C}$ species in the substrate, which influences the adsorption mechanism directly via the CSR's and indirectly via the interaction with the TM atom in the compound [1, 15, 16, 17].

In heterogeneous catalysis, linear correlations (BEP relations) between activation and adsorption energies play an important role [3]. Activation energies for $\mathrm{N}_{2}$ dissociation on some TMC(111) surfaces [27], calculated as the transition-state energy barriers $\left(E_{\mathrm{TS}}\right)$ by use of a fixed bond-length method, are presented in the inset of Fig. 3 as a function of the $\mathrm{N}$ adsorption energy. They show that the BEP relation holds for the TMC's, as expected from the linear correlation between the activation energies and $\varepsilon_{\mathrm{CCM}}$ (dashed line in Fig. 22). The result is expected to apply generally, as variations in activation and adsorption energies are governed by the same basic mechanism, which for TM's is the $d$-band model [3] and for TMC's the CCM [15, 16, 17, 24].

The linear dependence of both adsorption and activation energies on $\varepsilon_{\mathrm{CCM}}$ opens up the possibility to design 
the TMC substrates using the CCM to suit different catalytic reactions. This can be illustrated by the ammonia synthesis $\left(\mathrm{N}_{2}+3 \mathrm{H}_{2} \rightleftharpoons 2 \mathrm{NH}_{3}\right)$, which is a commonly used reaction to develop new concepts and ideas in catalysis 28. By using a slightly modified micro-kinetic model [29, 30] the calculated catalytic activities of the TMC's are found to order as a volcano curve with respect to the single descriptor $\varepsilon_{\mathrm{CCM}}$ [31].

The descriptor $\varepsilon_{\mathrm{CCM}}$ is derived here for TMC's but is expected to be directly applicable for understanding processes on other TM compound surfaces (and probably even on other surfaces) with SR's similar to the ones on the TMC(111) surface. Such SR's can appear on stable surfaces near defects, such as vacancies, steps, and adsorbed clusters. For example, experimental studies show that oxygen atoms replace carbon atoms on $\mathrm{TiC}(100)$ and $\mathrm{ZrC}(100)$ surfaces when exposed to $\mathrm{O}_{2}$ [14, 32]. Our calculations on an $\mathrm{O}$ atom adsorbed in a $\mathrm{C}$ vacancy site on the $\mathrm{TiC}(100)$ surface show the presence of a pronounced TMSR localized around the $\mathrm{C}$ vacancy. The calculated adsorption energy $(-8.71 \mathrm{eV})$ is close to the value on the $\mathrm{TiC}(111)$ surface $(-8.76 \mathrm{eV})$, to be compared with the value $(-4.96 \mathrm{eV})$ on the vacancy-free $\mathrm{TiC}(100)$ surface, where no TMSR is present. This illustrates the general importance of TMSR's for surface reactions.

Taken together, our results provide a framework for a systematic analysis of the catalytic activity of the TMC's. We have shown that by theoretical means it is possible to find a single descriptor, corroborated by experimental results on the electronic structure of the TMC surfaces [18], for atomic and molecular adsorption, as well as for a simple reaction on the TMC's. The found scaling and BEP relations provide simple and efficient connections between the (local) surface electronic structure and its reactivity, of importance for studying defect (e.g., vacancy) and ligand effects. There are thus implications for surfaces, nanosystems, and catalysis, and the introduced concepts should ramify to other systems and stimulate the development of similar models for other classes of materials.

Acknowledgments. The calculations were performed at HPC2N and NSC via the Swedish National Infrastructure for Computing. Support from the Swedish Research Council is acknowledged. B. I. Lundqvist gratefully acknowledges support from the Lundbeck foundation (Denmark) via the Center for Atomic-scale Materials Design.

* Corresponding author: alevoj@chalmers.se

[1] D. Nocera, Photosynth. Res. 91, 133 (2007).

[2] D. Nocera, Science 321, 1072 (2008).

[3] T. Bligaard et al., in Chemical Bonding at Surfaces and Interfaces, edited by A. Nilsson, L. G. M. Pettersson, and J. K. Nørskov, (Elsevier, Amsterdam, 2008).

[4] D. M. Newns, Phys. Rev. 178, 1123 (1969).
[5] B. I. Lundqvist et al., Surf. Sci. 89, 196 (1979).

[6] B. Hammer and J. K. Nørskov, Nature 376, 238 (1995).

[7] B. Hammer and J. K. Nørskov, Adv. Catal. 45, 71 (2000).

[8] J. Sehested et al., Top. Catal. 45, 9 (2007).

[9] F. Studt et al., Science 320, 1320 (2008).

[10] J. G. Chen, Chem. Rev. 96, 1477 (1996); H. H. Hwu and J. G. Chen, Chem. Rev. 105, 185 (2005) and references therein.

[11] J. R. Kitchin et al., Catal. Today 105, 66 (2005).

[12] E. M. Fernández et al., Angew. Chem. Int. Ed. 47, 4683 (2008).

[13] H. Toulhoat and P. Raybaud, J. Catal. 63, 216 (2003).

[14] F. Viñes et al., J. Chem. Phys. 122, 174709 (2005); F. Viñes et al., J. Phys. Chem. 111, 1307 (2007).

[15] C. Ruberto et al., Phys. Rev. B 75, 235438 (2007).

[16] A. Vojvodic et al., Surf. Sci. 600, 3619 (2006).

[17] C. Ruberto et al., Surf. Sci. 600, 1612 (2006); Solid State Commun. 141, 48 (2007).

[18] K. Edamoto et al., Phys. Rev. B 46, 7127 (1992); K. Edamoto, et al., Phys. Rev. B 43, 3871 (1991); T. Anazawa et al., Surf. Sci. 328, 263 (1995)

[19] To reduce the complexity and allow for trend studies, all considered TMC's are described in $\mathrm{NaCl}$ structure, which all except WC adopt in either stable or metastable phase.

[20] S. R. Bahn and K. W. Jacobsen, Comput. Sci. Eng. 4, 56 (2002); http://wiki.fysik.dtu.dk/dacapo.

[21] The spin-polarized approximation of the exchangecorrelation functional according to Perdew-Wang (PW91) 22] is employed. Ultra-soft pseudopotentials [33] and $400 \mathrm{eV}$ energy cutoff for the one-electron orbitals are used. The $k$-point sampling of the $3 \times 3 \times 8$ TMC(111) slabs is performed in a $(4,4,1)$ MonkhorstPack grid. Repeated slabs are separated by at least $10.8 \AA$ thick vacuum region. During structural relaxations, the two bottom atomic layers are constrained to the corresponding bulk geometry. Adsorption is allowed on one (the TM terminated) of the two slab surfaces in the fcc site (the stable site for adsorption on $\mathrm{TiC}$ [15]) and the electrostatic potential is adjusted accordingly [34]. The influence of higher coverage has been considered in Ref. [15], illustrating the dominance of direct adsorbate-substrate forces over lateral interactions.

[22] J. P. Perdew, K. Burke, and M. Ernzerhof, Phys. Rev. Lett. 77, 3865 (1996).

[23] The calculated TMC lattice parameters in the bulk $(\mathrm{NaCl})$ phase are $a=4.684,4.332,4.164,4.702,4.492$, 4.450, 4, 479, $4.382 \AA$ for $\mathrm{ScC}, \mathrm{TiC}, \mathrm{VC}, \mathrm{ZrC}, \mathrm{NbC}, \mathrm{MoC}$, TaC, WC, respectively.

[24] A. Vojvodic and C. Ruberto (unpublished).

[25] For the ligand systems with one, two and three $\mathrm{V}$ atoms we obtain $E_{\text {ads }}=-8.09,-7.78,-7.28 \mathrm{eV}$ and $\varepsilon_{\mathrm{CCM}}=$ $0.01,-0.08,-0.11 \mathrm{eV}$, respectively.

[26] F. Abild-Pedersen et al., Phys. Rev. Lett. 99, 016105 (2007).

[27] Owing to a strongly adsorbed molecular precursor, relevant activation energies are calculated with respect to the molecular adsorption energy.

[28] A. Hellman et al., J. Phys. Chem. B 110, 17719 (2006); M. Boudart, Top. Catal. 1, 405 (1994).

[29] It is assumed that $\mathrm{N}_{2}$ dissociation is the rate-determining step and that all other elementary steps are thus in quasiequilibrium [30]. The turnover frequency (TOF) is given by $\mathrm{TOF}=2 k P_{N_{2}} \theta_{*} \theta_{N_{2}}(1-\gamma)$, where $\gamma$ is the approach to equilibrium. The BEP and scaling relations are used 
when calculating the TOF as a function of $\varepsilon_{\mathrm{CCM}}$.

[30] K. Honkala et al., Science 307, 555 (2005).

[31] The ammonia synthesis is used just to prove the benefits of a single descriptor, not to suggest that TMC's are particularly good catalysts for it. Rather, reactions requiring a strong molecular precursor, for instance, oxidation of methane, are a more interesting group of reactions in a real application

[32] Y. Shirotori et al., Surf. Sci. 584, 237 (2005).

[33] D. Vanderbilt, Phys. Rev. B 41, R7892 (1990).

[34] L. Bengtsson, Phys. Rev. B 59, 12301 (1999). 